\title{
Out-of-hospital cardiac arrest: stay and play or scoop and run (to an ECMO centre)
}

Caleb M Fisher

"You may delay, but time will not."

Benjamin Franklin

Outcomes from those who have an out-of-hospital cardiac arrest $(\mathrm{OHCA})$ remain poor. This is despite greater community engagement in bystander cardiopulmonary resuscitation $(\mathrm{CPR})$, and improvements in the chain of survival with the recognition of benefits of early defibrillation, targeted temperature management and early revascularisation. For all of this, reported intact neurological survival remains low, ${ }^{1-3}$ with a clear association between poor neurological outcome and prolonged CPR and longer time to return of spontaneous circulation. ${ }^{4}$

Our improved understanding of the limitations of prolonged CPR and the diminishing benefit of the low flow organ perfusion it provides, coupled with improvements in extracorporeal membrane oxygenation (ECMO) technology, has led to increasing interest in the role and timing of ECMO in patients with OHCA. ${ }^{4}$ The provision of ECMO to this group of patients (now under the label of ECPR) is based on the practical and physiological principle that ECMO will provide better end-organ perfusion (especially for the brain) than conventional resuscitation algorithms and allow time for important definitive interventions and hence improve survival. In this regard, the evidence for the role of ECPR in OHCA has been evolving rapidly in the past decade, with several key research articles suggesting a significant benefit in terms of intact neurological outcomes by the application of timely, efficient and process-driven systems, incorporating ECPR into conventional resuscitation pathways. ${ }^{5-8}$

Important points raised by these studies relate to the importance of time from cardiac arrest to the initiation of ECPR and, correspondingly, the location of ECPR initiation. To this end, Bernard and colleagues ${ }^{9}$ have given us important insights into these questions in this edition of Critical Care and Resuscitation. In their study, Bernard et al conducted a retrospective review of OHCA patient outcomes with either ECPR or non-ECPR management in Melbourne, Australia. Over the period between January 2016 and December 2019, 223 OHCA patients meeting the inclusion criteria were transported either to the ECPR centre $(n=49 ; 22 \%)$ or to a non-ECPR centre $(n=174 ; 78 \%)$ respectively. Of the 23/49 patients who were able to be cannulated at the ECPR centre, survival was poor, with only four out of 23 patients $(17 \%)$ surviving to hospital discharge neurologically intact. Importantly, although this was higher than those admitted to non-ECPR centres, with three out of 174 patients (2\%) with a good neurological outcome on follow-up, there were significant and important differences between these groups. For example, the patients transported to the ECPR centre were younger, had higher rates of shockable rhythms, and were more likely to have had an OHCA in a public space. All these factors are well documented to improve outcomes of both OHCA and ECPR.

Of particular interest to the refinement of the role of ECPR in the management of OHCA was the time from initial call to arrival in the emergency department (ED) and to the commencement of ECMO flow. The reported median time for both groups to arrival in the ED was 63.9 minutes for the ECPR centre and 67.8 minutes for the non-ECPR centres. While the investigators were unable to retrieve data on time to ECMO commencement, other studies have reported this requires at least an additional 15-20 minutes after arrival in the ED, even in expert hands.6,10 That this total duration of time is significant for patient outcome in OHCA and ECPR may be evident by the reported mortality rate that can potentially be attributed to early profound ischaemic reperfusion injury ( $n=11 / 19,58 \%)$ and by the fact that ECPR was not even attempted in 13/20 patients at the ECPR centre. In contrast, a recent retrospective analysis of two large OHCA trials found that ECPR applied between 20 and 59 minutes after OHCA resulted in $47 \%$ neurological intact survival. ${ }^{8}$

So, what are the implications of the Melbourne findings for the management of OHCA? First, we must ensure that high quality CPR and application of international resuscitation algorithms remain the cornerstone of management. Second, we need robust randomised controlled trials applied in the Australian context, looking explicitly, via formal protocolised triggers (particularly in younger, less comorbid patients with refractory $\mathrm{OHCA}$ ), at the questions of:

- the location of ECPR and whether it can be efficiently applied in the field — research from Paris suggested that such an approach is feasible but significant challenges in meeting acceptable time frames to ECMO flow remain; ${ }^{11}$ and

- the role of protocolised early transfer to an ECPR centre, based on internationally implemented pathways - the 


\section{EDITORIAL}

challenges with this approach are evident by early results from the Extracorporeal Oxygenation for Refractory Cardiac Arrest (EROCA) trial suggesting that time to cannulation and ECMO flow within prescribed periods can be challenging. ${ }^{12}$

Despite these challenges, local and international research groups are actively attempting to provide insight to these questions with several trials currently underway. ${ }^{13-16}$ In addition, with all our enthusiasm for ECPR in this setting, we must remain mindful of how we utilise this intervention. ECPR in any format is a resource- and time-intensive endeavour, and the too wide an application of this approach may have a negative impact on the current well established OHCA chains of survival in place, as experienced by Alm and colleagues ${ }^{17}$ in Norway. Moreover, and especially given the results available to date, indiscriminate use may create an increased human and emotional burden on families, and any health care system struggling to adjust to the ongoing coronavirus disease 2019 (COVID-19) realities.

In conclusion, Bernard et al's findings highlight these challenges and offer the opportunity of identifying specific pathways to design further randomised controlled trials that will allow our collaborative ECMO community to identify the precise role and timing of ECPR in OHCA. However, for the moment, a "scoop and run approach" to OHCA requires much more robust evidence.

\section{Competing interests}

The author declares that he does not have any potential conflict of interest in relation to this manuscript.

\section{Author details}

Caleb M Fisher ${ }^{1,2}$

1 Department of Intensive Care, Austin Hospital, Melbourne, VIC, Australia.

2 Department of Critical Care, University of Melbourne, Melbourne, VIC, Australia.

Correspondence: caleb.fisher@austin.org.au

doi: https://doi.org/10.51893/2022.1.E

\section{References}

1 Virani SS, Alonso A, Benjamin EJ, et al. Heart disease and stroke statistics-2020 update: a report from the American Heart Association. Circulation 2020; 141: e139-596.

2 Gräsner JT, Lefering R, Koster RW, et al. EuReCa ONE-27 Nations, ONE Europe, ONE Registry: a prospective one month analysis of out-of-hospital cardiac arrest outcomes in 27 countries in Europe. Resuscitation 2016; 105: 188-95.

3 Schluep M, Gravesteijn BY, Stolker RJ, et al. One-year survival after in-hospital cardiac arrest: a systematic review and meta-analysis. Resuscitation 2018; 132: 90-100.
4 Goto Y, Funada A, Goto Y. Relationship between the duration of cardiopulmonary resuscitation and favorable neurological outcomes after out-of-hospital cardiac arrest: a prospective, nationwide, population-based cohort study. J Am Heart Assoc 2016; 5: e002819.

5 Richardson AS, Schmidt M, Bailey M, et al. ECMO cardio-pulmonary resuscitation (ECPR), trends in survival from an international multicentre cohort study over 12-years. Resuscitation 2017; 112: 34-40.

6 Yannopoulos D, Bartos J, Raveendran G, et al. Advanced reperfusion strategies for patients with out-of-hospital cardiac arrest and refractory ventricular fibrillation (ARREST): a phase 2, single centre, open-label, randomised controlled trial. Lancet 2020; 396: 1807-16.

7 Boros M. ACC 2021: the Prague OHCA study. Radcliffe Cardiology, 2021 https://www.radcliffecardiology.com/ACC\%2021-PragueOCHA-Study (viewed Dec 2021).

8 Bartos JA, Grunau B, Carlson C, et al. Improved survival with extracorporeal cardiopulmonary resuscitation despite progressive metabolic derangement associated with prolonged resuscitation. Circulation 2020; 141: 877-86.

9 Bernard SA, Hopkins SJ, Ball JC, et al. Outcomes of patients with refractory out-of-hospital cardiac arrest transported to an ECMO centre compared with transport to non-ECMO centres. Crit Care Resusc 2022; 24: 7-13.

10 Stub D, Bernard S, Pellegrino V, et al. Refractory cardiac arrest treated with mechanical CPR, hypothermia, ECMO and early reperfusion (the CHEER trial). Resuscitation 2015; 86: 88-94.

11 Lamhaut L, Hutin A, Puymirat E, et al. A pre-hospital extracorporeal cardio pulmonary resuscitation (ECPR) strategy for treatment of refractory out hospital cardiac arrest: an observational study and propensity analysis. Resuscitation 2017; 117: 109-17.

$12 \mathrm{HsuCH}$, Meurer WJ, Domeier R, et al. Extracorporeal Cardiopulmonary Resuscitation for Refractory Out-of-Hospital Cardiac Arrest (EROCA): results of a randomized feasibility trial of expedited out-of-hospital transport. Ann Emerg Med 2021; 78: 92-101.

13 NHMRC Clinical Trials Centre, University of Sydney. CPR, preHospital ECMO and Early Reperfusion (CHEER 3 Trial) for patients in refractory cardiac arrest to improve survival to hospital discharge [ACTRN12619000303145]. Australian New Zealand Clinical Trials Registry, 2021. https://www.anzctr.org.au/Trial/Registration/ TrialReview. aspx?id=376943 (viewed Dec 2021).

14 National Library of Medicine, US. A comparative study between a pre-hospital and an in-hospital circulatory support strategy (ECMO) in refractory cardiac arrest (APACAR2) [Identifier: NCT02527031]. ClinicalTrials.gov, 2021. https://clinicaltrials.gov/ct2/show/ NCT02527031 (viewed Dec 2021).

15 Singer B, Reynolds JC, Davies GE, et al. Sub30: Protocol for the Sub30 feasibility study of a pre-hospital extracorporeal membrane oxygenation (ECMO) capable advanced resuscitation team at achieving blood flow within 30 min in patients with refractory outof-hospital cardiac arrest. Resusc Plus 2020; 4: 100029.

16 NHMRC Clinical Trials Centre, University of Sydney. A randomised controlled trial on survival in out of hospital cardiac arrest patients treated with two existing transfer options: expedited transfer versus non-expedited transfer from scene to hospital - the EVIDENCE Study [ACTRN12619000303145]. Australian New Zealand Clinical Trials Registry, 2021. https://www.anzctr.org.au/Trial/Registration/ TrialReview. aspx?id=381456\&isReview=true (viewed Dec 2021).

17 Alm-Kruse K, Sørensen G, Osbakk SA, et al. Outcome in refractory out-of-hospital cardiac arrest before and after implementation of an ECPR protocol. Resuscitation 2021; 162: 35-42. 